\title{
Congenital bicuspid aortic valve: Steps toward providing an answer to the question of antibiotic endocarditis prophylaxis
}

\author{
Aaron M. Wolfson ${ }^{1}$, Jeanne M. DeCara², Marion A. Hofmann Bow man ${ }^{2}$ \\ 1. Department of Medicine, University of Chicago, Chicago, IL, USA. 2. Section of Cardiology, Department of Medicine, \\ University of Chicago, Chicago, IL, USA
}

Correspondence: Aaron M. Wolfson. Address: University of Chicago, 5841 S. Maryland Ave, MC6080, Chicago, IL 6063, USA. Email: wolfsonaaron@gmail.com or mhofmann@medicine.bsd.uchicago.edu

Received: J une 29, 2015

DOI : $10.5430 /$ crim.v2n3p73
Accepted: July 20, 2015

URL: http://dx.doi.org/10.5430/crim.v2n3p73

Online Published: July 27, 2015

\begin{abstract}
We report a case of congenital bicuspid aortic valve infective endocarditis in an 18-year-old male with no significant past medical history. He presented to the emergency department from an ophthalmology clinic with new right-eye vision changes. Initial EKG revealed sinus tachycardia and he was hemodynamically stable. Expedited diagnostic transesophageal echocardiography revealed vegetations on both aortic leaflets, an aortic root abscess, and a fistulous tract extending from the left ventricular outflow tract into the right ventricular outflow tract. Although he was evaluated by the cardiothoracic surgery team and scheduled for operative repair, he acutely became bradycardic, developed pulseless electrical activity, and was resuscitated on multiple occasions, but died suddenly from PEA arrest within 24 hours of presenting to the emergency department. This case demonstrates complications of acute bacterial endocarditis. We discuss the current debate surrounding the role of antibiotic prophylaxis for infective endocarditis. Additionally, we suggest that patients with a bicuspid aortic valve, a common congenital defect with a known increased risk for bacterial endocarditis, might represent a unique cohort to prospectively study whether antibiotic prophylaxis reduces the incidence, morbidity, and mortality of bacterial endocarditis in patients at increased risk.
\end{abstract}

\section{Keywords}

Infective endocarditis, Bicuspid aortic valve, Endocarditis prophylaxis

\section{I ntroduction}

The debate surrounding the role of antibiotic prophylaxis for infective endocarditis (IE) continues to be controversial, in part, because of the absence of randomized trial data ${ }^{[1,2]}$. As such, current IE guidelines are based on expert opinion from retrospective and observational studies ${ }^{[1,2]}$. One obvious obstacle for a definitive prospective randomized trial is the low incidence of disease (3-10 cases/100,000 person-years) ${ }^{[3]}$. Any prospective study evaluating IE prophylaxis would require a large sample size and long study duration to ensure adequate statistical power. A recent large, retrospective study by Dayer et al. ${ }^{[4]}$ highlights the necessity for such prospective evaluation. Their study identified a temporal association 
between an increase in the number of cases of bacterial endocarditis and the reduction in antibiotic prophylaxis prescriptions in England following the 2008 NICE guidelines ${ }^{[5]}$ recommending the complete cessation of prophylaxis.

With the above in mind, we discuss below why a prospective study of IE prophylaxis might be most prudent in patients with congenital bicuspid aortic valves (BAV); a patient sub-group that current data suggest are at a higher risk of developing native aortic valve endocarditis (NAVE) than might be expected based solely on the prevalence (0.2\%-2\%) ${ }^{[6-9]}$ of this congenital lesion. For example, in a study of 856 prospectively enrolled patients with NAVE, 16\% were found to have bicuspid valves ${ }^{[6]}$. Another study over a 29 year period found that $12.3 \%$ of 408 cases of NAVE occurred in individuals with a $\mathrm{BAV}^{[7]}$. In addition to this, patients with BAV IE were found to be younger, have fewer comorbidities, and a much higher frequency of perivalvular abscesses compared to patients with a normal tricuspid aortic valve ${ }^{[6]}$. Taken together, this evidence suggests that patients with BAV may be at higher risk of developing IE and be more likely to have significant adverse outcomes.

\section{Case presentation}

We report a case of congenital BAV IE in an 18-year-old male with no significant past medical history. He presented to the emergency department from an ophthalmology clinic with new right-eye vision changes and exam findings consistent with a retinal artery branch occlusion. He had normal blood pressure and was in no acute distress. The physical examination was notable for splinter hemorrhages and a cardiac murmur. The 12 lead electrocardiogram showed sinus tachycardia and was otherwise normal, although he had intermittent 1-3 minute long runs of supraventricular tachycardia (likely AVNRT) on telemetry monitoring, which terminated spontaneously. In light of his physical examination and several months of fever and weight loss, the presumptive diagnosis of endocarditis was made and subsequently confirmed echocardiographically. Despite an extensive history and physical exam, the patient lacked any identifiable risk factors or skin changes predisposing him to the development of bacteremia and subsequent endocarditis. Transesophageal echocardiography (TEE) revealed a bicuspid aortic valve with vegetations on both leaflets, an aortic root abscess (see Figure 1), and a fistulous tract extending from the left ventricular outflow tract into the right ventricular outflow tract (not shown). Immediately following his TEE he was transferred to the cardiac intensive care unit, evaluated by the cardiothoracic surgery team, and scheduled for operative repair. Before surgery he became acutely bradycardic, developed pulseless electrical activity, and was resuscitated on multiple occasions over a few hour span before finally expiring within 24 hours of hospital admission. An autopsy was performed and confirmed an acute endocarditis of a congenital BAV with a large perivalvular abscess with tissue loss (see Figure 2). Blood cultures drawn at admission came back positive for Streptooccus mitis.

Figure 1. Transesophageal echocardiogram

Note. Transesophageal echocardiogram with aortic valve vegetations and extensive abscess formation in the aortic root and atrioventricular septum. Left atrium-LA; left ventricle-LV; mitral valve-MV; ascending aortic-Ao; **-aortic valve vegetation; yellow arrow-abscess.

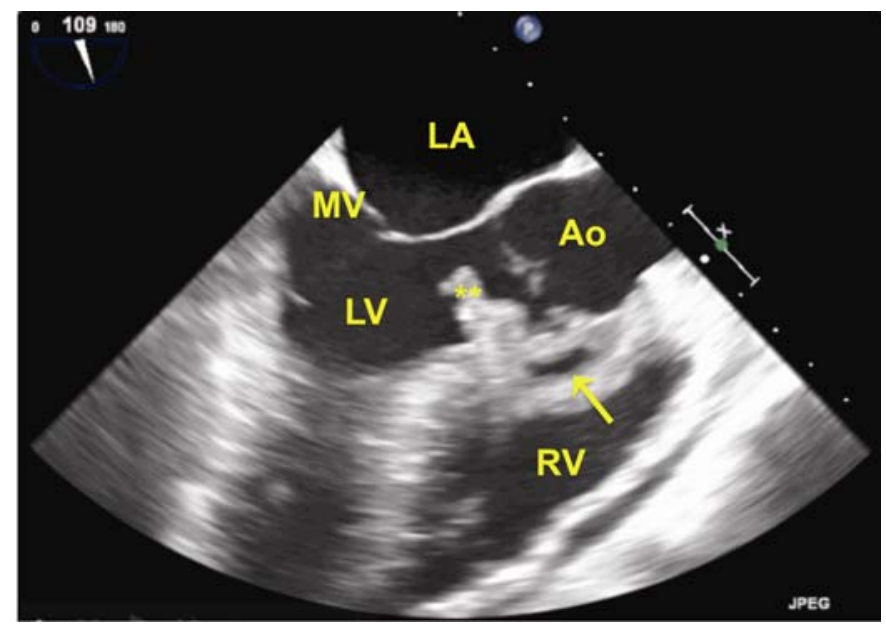


Figure 2. Autopsy specimen

Note. Autopsy confirming bicuspid aortic valve endocarditis with a fistulous tract extending from the left ventricular outflow tract into the right ventricle. Right ventricle-RV; ascending aorta-Ao; pulmonary artery-PA; mitral valve-MV; yellow arrow-fistulous tract.

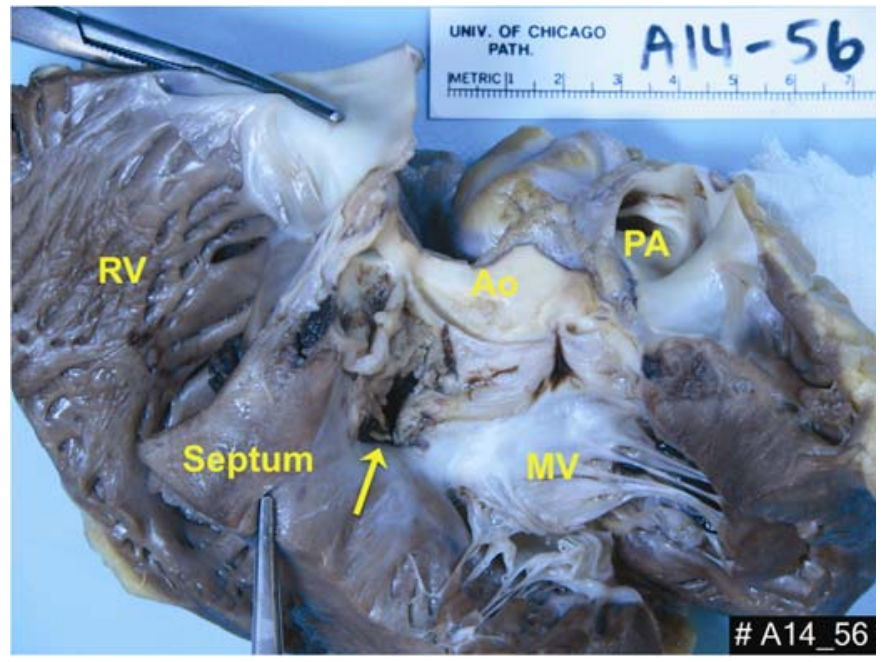

\section{Discussion}

This is an unfortunate case of infective endocarditis and likely could not have been prevented. Had the patient undergone a procedure for which IE prophylaxis might have been a consideration, current guidelines would not have supported such a decision. The 2007 American Heart Association guidelines on the prevention of infective endocarditis advocate that prophylactic antibiotics only be used in a subset of invasive dental procedures (those with greatest propensity for transient bacteremia) in patients at the highest risk of adverse outcomes from IE, but excludes those patients at high risk of acquisition of IE ${ }^{[10]}$. Similarly, the 2009 European Society of Cardiology guidelines recommend against widespread use of antibiotic prophylaxis and posit that prophylaxis be reserved only for the highest risk patients, including those at the highest incidence and/or the highest risk of adverse outcome from $\mathrm{IE}^{[3]}$. In these guidelines, congenital bicuspid aortic valves are not included as a high-risk population warranting antibiotic prophylaxis. Whether or not this population would benefit from antibiotic prophylaxis is unknown and we argue is important to answer given the high morbidity and mortality observed in patients with $\mathrm{BAV}^{[6,7]}$. Cardiovascular disorders represent the foremost cause of preventable death globally ${ }^{[11]}$. Given the high incidence of this congenital lesion in the general population $(0.2 \%-2 \%)$ together with a higher susceptibility for IE, we suggest that patients with BAV represent a unique cohort to prospectively study the role of antibiotic prophylaxis in reducing morbidity and mortality associated with IE.

In light of the current available evidence, or lack thereof, there is a clear need for a more deliberate evaluation of the role of antibiotic prophylaxis against infective endocarditis. At present, current data suggests that a prospective, randomized, placebo-controlled study in a population with congenital bicuspid aortic valves would provide a good framework to gain a greater understanding on this long-debated question.

\section{Conflict of interest}

The authors have no potential conflicts of interest to declare.

\section{References}

[1] Duval X, Hoen B. Prophylaxis for infective endocarditis: let's end the debate. The Lancet. 2014. http://dx.doi.org/10.1016/S0140-6736(14)62121-8

[2] Falces C, Miró JM. Prevention of infective endocarditis: between progress in scientific knowledge and the lack of randomized trials. Rev Esp Cardiol (Engl Ed). 2012; 65(12): 1072-4. http://dx.doi.org/10.1016/j.recesp.2012.06.020

[3] Habib G, Hoen B, Tornos P, et al. Guidelines on the prevention, diagnosis, and treatment of infective endocarditis (new version 2009). Eur Heart J. 2009; 30: 2369-2413. http://dx.doi.org/10.1093/eurheartj/ehp285 
[4] Dayer MJ, Jones S, Prendergast B, et al. Incidence of infective endocarditis in England, 2000-13: a secular trend, interrupted time-series analysis. The Lancet. 2015; 385(9974): 1219-28. http://dx.doi.org/10.1016/S0140-6736(14)62007-9

[5] Prophylaxis against infective endocarditis | Guidance and guidelines / NICE, (n.d.). http://www.nice.org.uk/guidance/cg064 (accessed January 29, 2015).

[6] Tribouilloy C, Rusinaru D, Sorel C, et al. Clinical characteristics and outcome of infective endocarditis in adults with bicuspid aortic valves: a multicentre observational study. Heart. 2010; 96: 1723-1729. http://dx.doi.org/10.1136/hrt.2009.189050

[7] Lamas CC, Eykyn SJ. Bicuspid Aortic Valve-A Silent Danger: Analysis of 50 Cases of Infective Endocarditis. Clin Infect Dis. 2000; 30: 336-341. http://dx.doi.org/10.1086/313646

[8] Siu SC, Silversides CK. Bicuspid Aortic Valve Disease. J Am Coll Cardiol. 2010; 55: 2789-2800. http://dx.doi.org/10.1016/j.jacc.2009.12.068

[9] Chandra S, Lang RM, Nicolarsen J, et al. Bicuspid Aortic Valve: Inter-racial difference in frequency and aortic dimensions: Impact of race in adult BAV in a single center retrospective observational study. JACC Cardiovasc Imaging. 2012; 5: 981-989. http://dx.doi.org/10.1016/j.jcmg.2012.07.008

[10] Wilson W, Taubert KA, Gewitz M, et al. Prevention of Infective Endocarditis Guidelines From the American Heart Association: A Guideline From the American Heart Association Rheumatic Fever, Endocarditis, and Kawasaki Disease Committee, Council on Cardiovascular Disease in the Young, and the Council on Clinical Cardiology, Council on Cardiovascular Surgery and Anesthesia, and the Quality of Care and Outcomes Research Interdisciplinary Working Group. Circulation. 2007; 116: 1736-1754. http://dx.doi.org/10.1161/CIRCULATIONAHA.106.183095

[11] Santulli G. Epidemiology of cardiovascular disease in the 21st century: updated numbers and updated facts. JCvD. 2013 ; 1(1)1-2. 